compression was relaxed, and so the blood and bichromate were washed into the kidney. By either of those methods a more or less concentrated dose of the bichromate is sent into one kidney, but the poison is freely diluted by the general blood stream before it reaches the opposite kidney and thus it is possible to produce very marked renal lesions on the one side without the general health of the animal in any way suffering owing to the poison being too dilute to produce any marked effects on the opposite kidney. The injection of bichromate by this method is not followed by any symptoms of illness or discomfort and the only marked effect produced is a slight albuminuria. It is interesting to observe that the amount of albumin is always small and frequently not more than a trace. The animals remained in good health and were killed at intervals varying from two to eight months. The kidney on examination was found to be shrunken and the renal artery pervious. On microscopical examination the epithelium of the tubule was found to be shed over large areas of the kidney and the glomeruli also presented some changes such as shrinkage and atrophy. The great diminution in the size of the kidney was dependent, however, on the shedding of the tubular epithelium and the point of interest rested in the fact that notwithstanding the great diminution in the size of the kidney there was no overgrowth of interstitial tissue.

These experiments would seem to show that destruction of the renal epithelium by a metallic poison like bichromate of potash is not at any rate necessarily followed by any consecutive fibrosis. When a dose of bichromate equal to that used in the injections into the renal artery was injected into the general circulation through the jugular vein a destructive action on the cells of the renal tubules in both kidneys was produced resulting in practically total necrosis of the tubular epithelium. In such animals the excretion of urine was arrested and effects somewhat similar to those seen after complete nephrectomy were observed but vomiting and diarrhoea were more marked symptoms. A curious result is the fact that in the earlier stages of the action of this renal poison the albuminuria produced is by no means intense and in that respect offers a great contrast to the intense albuminuria seen clinically in many forms of acute diffuse nephritis. In fact. it would seem that very complete destruction of the epithelium of the tubules is possible without causing more than a slight albuminuria. In the other experiments just alluded to, where destruction of the epithelium of the tubules and atrophy of the kidneys were produced by temporary ligature of the ureter, the albuminuria observed in the secretion of the damaged kidney when the ureter was drained was also slight in amount, notwithstanding that the tubular and even the glomerular changes were very marked. The amount of albumin found in the urine in both these experimental conditions was much more comparable to that seen in the true granular kidney than to that present in the ordinary forms of Bright's disease, acute or chronic. It is, of course, quite possible that in both the experimental series the albuminuria was associated with the changes produced by the poison or by the ligature of the ureter on the glomeruli rather than on the tubular changes. The point to which I wish to direct attention at the present moment is that very extensive damage may be inflicted on the tubular epithelium without causing any large amount of albuminuria. This would suggest that albuminuria might normally be dependent either on glomerular changes or else that the epithelium of the tubules is to a certain extent necessary for its presence and that when this is more or less completely destroyed albuminuria is not such a marked phenomenon. The experimental results were sonewhat similar to those seen in advanced stages of granular kidney where large areas of the renal tubules are either destitute of epithelium or are merely lined by a layer of glass-like hyaline cells which have completely lost their characteristic renal structure.

A great deal more work is necessary in order to elucidate the action of renal poisons on the kidney and especially to determine whether the overgrowth of fibrous tissue that is such a constant accompaniment of chronic renal disease in almost all its forms is not really due to the long-continued or repeated action of toxic agents. It would certainly appear that the mere destruction of the higher elements of the kidney structure produced by the immediate action of a poison is not of itself sufficient to cause the fibroid overgrowth.

Sufficient has been said to show that a very considerable number of renal poisons exist capable of producing marked structural alterations in the organs. It will be noticed, however, that none of these poisons produce a clinical picture at all similar to that seen in Bright's disease, since one of the most conspicuous features in Bright's diseaseviz., dropsy - is absent. Varying degrees of nephritis may be produced experimentally from one where but a slight albuminuria exists to another where complete suppression of urine with uræmic manifestations and rapid death occurs, but in none of these is dropsy seen. Although at first sight this would seem to mark a clear difference between the experimental and the clinical facts further analyses show really that there is considerable resemblance between the experimental and the clinical phenomena. Some of the most severe forms of acute nephritis seen in the human subject are not accompanied by dropsy and not only is this the case with regard to forms of very acute nephritis of uncertain etiology of obscure origin but the same thing may even be seen in such a well-known and definite nephritis as that seen as a complication of scarlet fever. Every physician has seen cases of intense nephritis with hæmaturia, albuminuria, \&c., followed shortly by arrest of the renal secretion and the onset of marked uræmic symptoms not uncommonly followed by death, and yet notwithstanding the intensity of the renal lesion no anasarca has been present. In many of these cases the uræmic symptoms are well marked and are of a somewhat similar character to those seen in the acute uræmia complicating Bright's disease. But in others-and this seems to me a point of considerable theoretical interest-the phenomena are more closely similar to those seen in so-called obstructive suppression. In other words, in some cases an intense inflammatory affection of the kidneys may occur, associated with complete suppression of urine often lasting for many days, and the patient will succumb with symptoms precisely similar to those seen in calculous obstruction and known as latent uræmia. This group of cases in the human subject is no doubt somewhat rare but still it occurs and the results are very similar if not identical with the phenomena produced in the laboratory by the action of poisons suddenly destroying the integrity of the kidney.

\section{POST-GRADUATE DEMONSTRATION ON RHEUMATOID ARTHRITIS.}

By CHARLES J. MACALISTER, M.D. EDIN.,

\section{M.R.O.P. LOND.,}

LECTURER ON CLINICAL MEDICTNE, UNIVERSITY OF LIVERPOOL;

PHYSICIAN TO THE IIVERPOOL ROYAL SOUTHERN HOSPITAL AND

TO THE COUNTRY HOSPITAL FOR CHILDREN; SENIOR MEDICAI OFFICER TO THE HOME FOR IYCUBABIFS.

Ix a general hospital we have little opportunity for studying the disease which is known as chronic rheumatoid arthritis because, being regarded as an incurable or very chronic complaint, the tendency is to palliate suffering for a time by rest and nursing and good feeding and by a somewhat routine administration of drugs and when the time limit has expired, or sooner, the patients are discharged in conditions of health not essentially different from those in which we found them. In a home for incurables, on the other hand, the patients are under observation for many years and it becomes possible to work at the disease with reference to its causes, course, and treatment in a way which would otherwise be impossible. The study of this malady in its chronic forms throws light upon certain cases, however, which we come across in the hospital wards and when their records are compared they reflect upon one another in a way which goes to prove that rheumatoid arthritis is a very complex disorder which has many factors concerned in its etiology. The disease as we frequently see it is in an advanced stage, often with very little activity about it or even cured, not a few cases reaching the stage of cure after numerous joints have been destroyed; and when this is the case all that remains to be done is to promote mobility in the parts which often become wonderfully useful even when great deformity has been brought about. It is astonishing to witness the dexterity with which a woman whose metacarpo-phalangeal joints are all dislocated, so that the fingers hang like so many tassels, can knit and sew 
and the amount of power which she acquires when pain disappears and stiffness of the larger joints has been lessener by passive movement or hot air and baths, and happy may patients consider themselves if this inactive stage is reached, for unfortunately another aspect of the disease is very gruesome, where after prolonged suffering its final stages are unrelieved of pain and every movement is associated with torture. The sufferer then sits or lies in a condition of rigidity, the extremities wooden in their appearance, the lower ones presenting the solid cedema characteristic of the complaint when it assumes this form. It was formerly customary, following the teaching of Charcot, to classify cases accorcing' to their anatomical features in the types of flexion and of extension, but the type of deformity is not of great moment, the cause of the disease being the essential consideration, for if this can be discovered and dealt with it may be possible to arrest it either before it has produced much structural change or after crippling and deformity have been brought about, if the condition still remains active and progressive. I would at this point only warn you against the temptation to attempt the forcible extension of, or surgical interference with, joints in which the disease is active, for I have seen such proceedings to be followed by most unfortunate results and by the losing of ground which has been with difficulty gained.

The first case I show you illustrates a very chronic and progressive type of the disease, the patient being a woman, aged 67 years, whose illness began in 1885 with what she describes as an attack of "rheumatic fever" which lasted for six weeks or more. It involved all the joints, both great and small, and at length when the fever subsided recovery was not complete in the hand, which remained swollen and painful and the wrists and fingers gradually became contracted. Some time later the knees became affected, first of all swollen and finally contracted, and so in gradual succession over a long period of years the shoulders, the elbows, the hips, the ankles, and the feet have been similarly involved, the cervical and temporo-maxillary joints and even the synostoses of the skull not escaping, and now she is rigidly locked up in all her joints, unable to move a finger to help herself and suffering severe pains every time she is moved.

In connexion with this case I wish to direct particular attention to the history of an initial attack of rheumatic fever of long duration and to remind you that in our practice we every now and then come across cases very like rheumatio fever, presenting the painful, inflamed, and swollen joints, fever and sweatings, but differing from it in there being no liability to cardiac complications and in a tendency for chronic joint troubles to remain. I have records of a number of these cases and have pointed out ${ }^{1}$ that they represent the cases of rheumatic fever in which the salicylates are powerless, the fact being that they are not cases of rheumatic fever at all but are due to a different toxin which, if uneliminated, will lead to chronic rheumatoid arthritis. I consider the salicylates to be tests for rheumatic fever for which they are specifics and that if they produce no benefit the disease is rheumatoid and not rheumatism. I regard it as of great importance that these rheumatoid fevers should be recognised and treated energetically in view of the very serious consequences which may follow in their train. The toxins which give rise to them are generated in the economy, generally in association with some mucous surface, as the following case well illustrates.

A man, aged 26 years, was admitted to the Liverpool Royal Southern Hospital on July 31st, 1901, suffering from headache, profuse sour-smelling perspirations, and from pain, swelling, and slight redness of the knees, the elbows, and of the finger and toe-joints. There were miliaria all over the chest and abdomen, the tongue was thickly furred, and the bowels were constipated; the respiratory and circulatory systems were normal. He had been ill for seven days. The temperature ranged from $99 \cdot 6^{\circ} \mathrm{F}$. in the mornings to $101 \cdot 2^{\circ}$ in the evenings and neither this nor his general condition was improved by the administration of 10 grains of salicylate of sodium which was given every three hours for a week ; in fact, on August 5th, 6th, and 7th the temperature had become higher each night and the trouble had extended to the sterno-clavicular articulations. On the 7th guaiacol carbonate was substituted for the salicylate but on the 10th the temperature was still high and the left temporomaxillary articulation had become involved. There were

1 Royal Southern Hospital Reports, 1901. profuse perspirations and the skin was covered with miliaria. On this date it was observed that the secretions from the nose were blood-stained and it was at once directed that it should be washed ont several times daily with a lotion containing one drachm of liquor hamamelidis to one ounce of a saturatel solution of boric acid. The guaiacol was stopped and quinine was given in its place. On the following day (the 11th) the temperature was subnormal except in the evening, when it reached $99 \cdot 6^{\circ}$, and on and after the 12 th it remained normal. The pain rapidly subsided and he quickly improved. This man had a chronic rhinitis, which is a very frequent antecedent of the disease, and I would wish to impress the fact that it is not necessary to have ulceration in the nose (although I have seen it several times). It is quite sufficient to have a swollen mucosa the surfaces of which are in contact and $I$ have no doubt that the retained secretions undergo some infection which, being absorbed, gives rise to the trouble. I mention this case only by way of example, because although the nose is an obvious seat of absorption it occurs from many other places and I have seen it in pulmonary conditions, as in bronchiectasis with retained secretions, and in not a few cases it has occurred from some part of the alimentary canal. The majority of sufferers are women and the catarrhal affections of the genital tract are probably responsible for the disease in many, but there are other factors to consider in this relation to which I shall refer later.

There was a case admitted into the Liverpool Royal Southern Hospital on Sept. 18th, 1901, which was of great interest because it led me to think about the way in which remedies seem to act in some cases of toxæmia. The man, aged 40 years, who had previously been quite healthy, had for 14 days been suffering from fever with pains and swellings of the joints of both his upper and lower extremities, and on admission he looked like a case of acute rheumatism. He was sweating profusely and the knuckles of both hands and the finger-joints were swollen and tender. The wrists, the shoulders, the elbows, and the ankles were very painful. The heart sounds were all closed, the lungs were normal, and the urine was of specific gravity 1018, acid, and contained no albumin. For seven days he was given salicylate of sodium (15 grains) every four hours, with the result that the temperature, which was remittent in type (from $99 \cdot 0^{\circ}$ A.M. to $101^{\circ}$ P.M.), became higher. Guaiacol was then tried without any benefit for three days and then, on Sept. 28th, hydrochloride of quinine was given, at first twice and afterwards three times daily, with the result that the temperature fell by a lysis extending over two days and his pains and swellings quickly disappeared. As in all these cases this patient perspired profusely, the sweat being soursmelling, but immediately the quinine was commenced it altered its character entirely and he began to eliminate from his skin a volatile matter having an odour more like garlic than anything else. This elimination continued for several days and then ceased. It was so offensive that he had to be removed from the ward the atmosphere of which became permeated with it. The recovery of the patient corresponded with the getting rid of this odorous material which was certainly connected with his complaint.

In connexion with this case let me remind you of one of another disease which I published in THE LANCET of Dec. 7th, 1895, p. 1428. In a case of profound uræmic coma, in which other remedies had been tried unavailingly, oxygen was administered through a tube inserted in one of the nostrils, with the result that the man speedily regained consciousness and ultimately recovered completely. Uræmia, as is well known, depends in many cases upon the fact that the poisons which cause it are incapable of stimulating the kidney to remove them. The presence of urea in the blood. stimulates the kidney and causes more blood to pass through it until the waste product is removed, but some of the poisons which cause uræmia have not this stimulating action on the organ and consequently they are retained and increase and cause narcosis, or convulsions. according to their nature, and in the case which I have referred to it seems reasonable to suppose that the oxygen changed the nature of the poison and rendered it less noxious and capable of elimination. Similarly in the case of rheumatoid fever which I have quoted, although the patient perspired profusely and passed urine which was not perceptibly abnormal, the poison was retained until quinine was given, when its nature was changed and it was freely eliminated. I believe that in many toxic diseases the remedies may act in this way; 
colchicum, for instance, in gout (which is only another example of a toxæmia which sometimes has manifestations very like those of rheumatoid arthritis), salicylates in acute rheumatism, and so forth; the drugs acting as vehicles for the poisons and rendering them capable of stimulating the kidneys or skin to remove them. When these rheumatoid fevers instead of recovering completely lead to chronic rheumatoid arthritis it seems probable that the poison either continues to be generated from its unascertained source, or that it produces perversions in the economy which we do not fully nnderstand, but even if it has existed for years and is still active the administration of quinine and the local treatment of diseased mucous surfaces will often do good as $I$ have repeatedly been able to demonstrate.

The next case to which I wish to invite your attention is interesting because it shows how the disease may be set up by septic fevers. Here it took its origin in a woman, now aged 30 years, in an attack of scarlet fever five years ago which was followed by the so-called scarlatinal rheumatism. This lasted for nine weeks, then came an interval of comparative freedom lasting four months, and this was succeeded by a febrile relapse with joint pains which kept her in bed for 18 weeks. She subsequently returned to her duty as a nurse for a few months, but the typical chronic rheumatoid form of disease then began in the first interphalangeal joint of the right hand and since then joint after joint has become implicated, until she is now bedridden and crippled, many of the joints having become distorted, her hands, feet, knees, \&c., presenting the usual deformities When recovering from the scarlet fever this patient noticed that her fingers used to become numb and dead at the ends and the feet were similarly affected and during the course of the disease, especially in the first five months, she had suffered severely from cramps which began in the upper intercostal muscles and later affected those of the thighs and legs. It is very common to find these two latter symptoms in cases of rheumatoid arthritis, but sometimes they both exist not only in the course of the disease when the joints are involved, but as antecedents to their implication and, taken in conjunction with the increased reflexes which are almost invariably present, they indicate that the toxin acts upon the nervous centres and renders them very excitable. My colleague, Dr. T. R. W. Armour, has found that by putting the limbs into splints, so that the irritation from the painful joints is lessened, the cramps have been relieved. If you feel this patient's hands and feet you will observe that they are cold and covered with moisture but under treatment with large doses of quinine she has improved wonderfully and I hope will reach the stage of arrest with deformity before long. Her thyroid gland is small and she has been taking thyroid extract for reasons which I shall refer to in my next case.

The chronic rheumatoid condition in this case has run a course very similar to those of plenty of others in which there has been no antecedent scarlet fever. We cannot always trace the source of the poison which causes it and there can be no doubt that it may originate in a great many different ways. In one girl here the disease started with absolute suddenness, a pain striking her under the knee one morning shortly after her breakfast and since then many joints have become involved and she is crippled, although only 15 years of age. In other cases the process has been less acute and longer intervals have existed between the joint invasions but the results are the same in the end with the difference that arrest of the disease takes place in some while in others it remains ever progressive and active. We cannot find the source of the toxin in every case but it should always be looked for and success will in some cases reward our efforts.

I am now going to show you a case which is very different from any which we have so far looked at. It is a unique case and has been of great interest to me because it has led my thoughts into channels which diverted me from the groove into which one is apt to get when a number of cases more or less resembling one another come under observation. The patient was 62 years of age when ardmitted to the home in 1891 and for three years she had suffered from rheumatoid symptoms which, commencing in the right wrist, rather rapidly affected in succession most of the other jo:nts, including those of her hands and fingers. She was entirely crippled when admitted and the disease was still active, manifesting itself not only in the synovial swellings round the joints but also in the intensely painful nodules which appeared from time to time on the aponeurosis of the occipitofrontalis. Shortly after admission to the home this patient had an apoplectic attack causing a right-sided hemiplegia, from which she recovered, and the greater distortion in the joints of the right hand are due to contractions following the stroke. Shortly after this seizure came a new and very extraordinary train of events not easy of explanation, for a gradual swelling took place in her limbs and trunk, but especially in the lower extremities, until at lengt $\bar{n}$ she had the appearance of a case of general anasarca. The trunk and upper extremities were only moderately swollen, but the lower extremities became enormous, the calves measuring 22 inches in circumference. There were no albumin and no evidence of cardiac or pulmonary disease to account for the condition. She was in a state of great mental depression, wept frequently and took not the slightest interest in her surroundings. The condition of the feet and legs was so remarkable, their proportions so enormous compared with other parts, that $I$ am afraid my attention was diverted from the true nature of this case and I regarded it for a long time as due to the solid cedema with which we are familiar in cases of rheumatoid arthritis, and it was only the course run by an ulcer which appeared on the dorsum of the right foot which led to a true interpretation of the case. This ulcer caused me much concern when it first appeared, because deep ulcers appearing in this disease are of very grave significance; we have had seven of them in the past 15 years, two on the knee, three in the dorsum of the foot, and two over the sacrum, and in every case they have been related to the death of the patient. In this case, however, the ulcer was evidently not an absorbing surface; it was indolent, painful, and there was not much discharge from it; it lasted for weeks and nothing would induce it to heal; its edges were precipitous, so that it presented the appearance of having been gouged out with a large cork borer. Looking at it one day and wondering what could be done to stimulate it and to promote some activity in the process of repair it seemed to me that its walls looked rather like mucoid tissue, and it was only then that it occurred to me that the case, whatever its primary nature, had myxcedema complicating it. She was at once put upon thyroid gland in gradually increasing doses until she was taking 15 grains daily in tabloid form. 'The result of this treatment was most satisfactory, the ulcer quickly healed, the swelling of the arms and trunk vanished and that of the legs diminished, so that the calves measured only 14 inches instead of 22 inches. With this the mental depression improved and she became quite cheerful and in addition the joints became less painful and swollen and in some cases more mobile. The feet and legs have never entirely returned to their proper shape and the residual swelling may be regarded as a local myxœdema together with the solid cedema which is so common in the disease.

I have told you that this case has influenced my impressions considerably with reference to some examples of rheumatoid arthritis and subsequent experience has taught me that there is a relationship between some manifestations of this disease and the thyroid functions. I have recently had two very striking cases illustrative of this fact, one patient being a woman, aged 43 years, who gave the following history. She had been a delicate girl and had suffered much from dyspepsia and neuralgia and from what she described "a sluggish circulation causing chilblains, coldness, and numbness of the hands." When aged 21 years, and for several years afterwards, she had frequent attacks of cramp in the hands, feet, and calves. The thumbs became forcibly flexed into the palms from time to time and there were twitchings of the muscles which became very easily fatigued. She had frequent nervous headaches, causing great prostration. About the same time, without any external swelling, the joints began to creak and the skin became dry, rough, and irritable during the winter but perspired freely during the summer. Her hair became weak and was constantly falling out and her singing voice, previously of good quality. was lost. The rheumatism of the joints never left her and although able to go about she suffered constantly, the trouble being increased by eczema which attacked her at 38 years of age. A year later the breast-bone began to be tender and the sterno-clavicular articulations became swollen and very tender. At 40 years of age she had a severe exacerbation with increased joint pains and muscular cramps. In the following three years there were further attacks with implication of the ankles and when I saw her early this year she presented the usual signs of this type of rheumatoid arthritis, the fingers being spindled and painful; there were stiffness and swelling in the knees and other 
joints ; the breast-bone, sterno-chondral junctions, and sterno-clavicular articulations were swollen and tender, and the lower extremities were in a state of solid cedema. I treated her with the pharmacopœial liquor thyroidei in conjunction with nitrite of sodium (her surface and extremities being very cold and numb) and the result has been most satisfactory, the swellings and pains having left her legs and hands. She now states that she is in better health than she has been for five years.

The other case to which I have referred was that of a young woman who in the spring of 1903 began to have severe rheumatic pains in the knees which became stiff and creaking. Later other large joints became similarly affected and the fingers became swollen and painful. She had for long been subject to coldness of the hands and feet, her fingers becoming dead white and numb, even in warm weather. When I saw her last August she was lame and stiff and there were peri-articular swellings of the fingers and wrists. The extremities were cold and sweating freely. Like the previous patient she suffered from severe nervous headaches which usually lasted for about a day. Her general health was good but she felt depressed and disinclined for work. This patient was treated with five grains of thyroid gland (tabloid) daily with the result that in three weeks the pains were gone and the swellings had vanished and she has had no relapse.

I have published elsewhere ${ }^{2}$ my views concerning the relationship which exists between certain cases of rheumatoid arthritis and Raynaud's disease and have referred to the coldness and numbness of the extremities which constitute early symptoms of rheumatoid arthritis as in the above two cases. You will find that migraine is another example of an associated condition and in some cases asthmatic attacks from time to time accompany fresh exacerbations of the rheumatoid pains. In some families you will find rheumatoid arthritis affecting one girl and Raynaud's disease or some modification of it present in her sisters; indeed, the association of the two conditions in a family or in the same individual is so frequent that it cannot be ignored. And so with the cramps they are not accidental to the disease, but are connected with it and symptomatic of the excited condition of the spinal centres to which the toxin gives rise.

I must refer you to $\mathrm{my}$ former papers 'on this subject for further consideration of the relationships which exist between rheumatoid arthritis and some other diseases.

Liverpool.

\section{CASES OF TOTAL ENUCLEATION OF THE PROSTATE FOR RADICAL} CURE OF ENLARGEMENT OF THAT ORGAN.

BY P. J. FREYER, M.A., M.D., M.CH.,

SURGEON TO KING EDWARD VII.'s HOSPITAL FOR OFFICERS AND TO ST. PETER'S HOSPITAL FOR STONE AND OTHER URINARY DISFASES, LONDON.

IN a series of lectures delivered by me at the Medical Graduates' College and Polyclinic in November, 1900, and published in THE LANCET of Jan. 12th (p. 79) and 19th (p. 149), 1901, I endeavoured to give a practical summary of our knowledge, as it then existed, of the disease generally known as "hypertrophy of the prostate," describing its pathology, symptoms, diagnosis, and various methods of treatment. It was then pointed out that there was, perhaps, no other disease in the whole range of surgery for which so many and diverse modes of treatment had been advocated, a fact in itself suggestive of the unsatisfactory nature of most if not all of them, so that at the time catheterism pure and simple, with all its disadvantages and dangers, reigned supreme in the practice of most surgeons as the least objectionable of all.

From time to time various procedures had been proposed and practised with a view to an attempt at radical cure of the disease. Most prominent amongst these may be mentioned partial prostatectomy (whether by the urethral, perineal, or suprapubic route), castration, vasectomy, and
Botini's operation, which consisted in an attempt to burn away by the electric cautery the so-called "middle" lobe of the prostate. Each of these procedures enjoyed a temporary though transient notoriety. I ventured to hold that partial prostatectomy by the suprapubic route, first performed by Belfield of America but best known in this country in connexion with the name of McGill who brought it prominently before the profession in 1888 , was the most practical attempt at a rational method of dealing with the obstruction caused by the enlarged prostate. The operation consisted in opening the bladder suprapubically and removing the prominent portions of the prostate in that viscus or as much of it as possible by means of scissors, forceps, and scoops of kinds. I myself had performed this operation on several occasions with some measure of success. But apart from the high mortality attending the procedure it possessed the disadvantage that, though frequently followed by the subsiclence of the most prominent symptoms, temporarily at least, and rendering the employment of the catheter more easy, in a very large proportion of cases the bladder failed to regain its power of expelling the urine. This was due to the fact that the outgrowth in the blarder is, as a rule, not the only or, indeed, the chief cause of the obstruction, as was imagined, which is mainly due to the lateral pressure on the urethra by the enlarged prostatic lobes. Indeed, when once the bladder had completely lost its expulsive powerthat is to say, when the whole of the urine had to be drawn off by the catheter-McGill's operation was practically incapable of restoring that power. Add to this the fact that, as only the prominent portions of the prostate in the bladder were removed, there was no immunity against recurrent outgrowth or general enlargement of the gland and we realise in what a very limited sense this operation could be regarded as radical. Owing to these considerations the operation, after enjoying a temporary and fitful notoriety for a few years, may be said to have died out of surgical practice. It was replaced, first by castration and later by vasectomy, which was practised extensively during the closing years of last century. Experience has shown that both these procedures were practically useless and the former dangerous. They have now passed into oblivion.

Shortly after these lectures were delivered-indeed, before they were published-on Dec. 1st, 1900, I performed a new, and what seemed at first sight a very formidable operation for radical cure of the disease-namely, total extirpation of the enlarged prostate. In a lecture delivered at the Medical Graduates' College in June, 1901, I gave full details of this and three further cases in which I had undertaken the operation, in all four with complete success. Two of the patients were shown at the lecture in perfect health, able to retain and pass their urine as well as ever they did, though previously completely dependent on the catheter. The lecture was published in July, 1901, and the operation was thus submitted for the consideration of the profession at large. The complete success that followed these operations entirely revolutionised my views regarding the treatment of this widespread and painful malady and, I submit, opened up a new era in this branch of surgery. Since then I have from time to time published lectures and papers on several series of cases of my operation. In this manner full details of my first 73 cases have been placed before the profession. Having now performed the operation in more than 100 cases I propose in this paper to review its present position, giving the latest details of the procedure and describing the anatomical and pathological considerations that render it practicable.

A careful examination of the specimens removed in these operations throws an entirely new light on the anatomy of the prostate and its relations to the surrounding structures, and shows that the descriptions contained in the anatomical text-books generally are incomplete and erroneous in treating that organ as a single body with a canal tunnelled tbrovigh it in the form of the prostatic urethra. The prostate is in reality composed of twin organs, of apparently purely ses u $u$ function, which in some of the lower animals remain dist no and separate throughout life, as they exist in the bun an male during the first four months of fotal existence. After that period, in the human fœtus, ther approach each other, and their inner aspects become agglutinated together, except along the course of the urethra, which they $\mathrm{n}$ nvelop in their embrace. These two glandular organs, which constitute the lateral lobes of the prostate, lhough welded toother, as it 